\title{
The Ground State of a Large Number of Particles on a Frozen Topography
}

\author{
A. Travesset \\ Physics Department, Iowa State University and Ames Lab. \\ Ames, IA, 50011, USA
}

\begin{abstract}
Problems consisting in finding the ground state of particles interacting with a given potential constrained to move on a particular geometry are surprisingly difficult. Explicit solutions have been found for small number of particles by the use of numerical methods in some particular cases such as particles on the sphere and to a much lesser extent on a torus. In this paper we propose a general solution to the problem in the opposite limit of very large number of particles $M$ by expressing the energy as an expansion in $M$ whose coefficients can be minimized by a geometrical ansatz. The solution is remarkably universal with respect to the geometry and the interaction potential. Explicit solutions for the sphere and the torus are provided. The paper concludes with several predictions that could be verified by further theoretical or numerical work.
\end{abstract}

PACS numbers: 61.72.Lk,61.30.Jf,61.72.Mm,62.20.Dc

\section{INTRODUCTION}

Problems related to determining optimal particle distributions under constraints are ubiquitous in the traditional sciences and have been under intense scrutiny in the mathematical community [1, 2]. Two of the many interesting examples are the determination of ground states of $M$ particles constrained to move on the sphere and interacting with a Coulomb potential, so called Thomson problem [3], whose more direct representation are classical electrons on helium bubbles 4] and the crystallization of particles on spheres, relevant for understanding the structure of PMMA beads on oil/water droplets [5]. Similar problems on more general geometries such as the torus or negative curvature surfaces are also of great experimental and theoretical interest [6, 7, 8].

Theoretical investigations are surprisingly difficult. Extensive numerical results obtained in the Thomson problem, 9, 10, 11], for example, show that the number of metastable states grow very fast with the total number of particles, preventing a numerical solution to the problem even for a number of particles of a few hundred particles. For problems on the sphere, a few rigorous analytical results and conjectures on the energy of the ground state for large number of particles exist [2, 8], but a description of the structure of these ground states, including practical tools on how to find them as well as its generalization to any given arbitrary geometry remains a completely open problem.

Recently, it has been shown that elasticity theory 6, 12, 13, 14] (see also 15, 16]) provides a powerful framework to discuss best particle configurations on spheres, which can then be easily generalized to deal with any arbitrary geometry. Building on these results, we propose a general solution for the structure of ground states on arbitrary geometries in the limit of large number of particles, and we construct the explicit solution for the case of a sphere and a torus. The solution is universal, in the sense that it applies for short-ranged poten- tials and, in some situations, for long-ranged potentials as well.

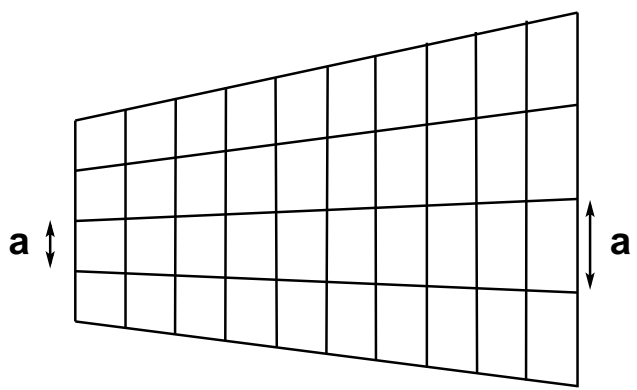

FIG. 1: Typical distortions due to geometric constraints on a perfect square lattice. If an additional row of atoms somewhere in the middle of the crystal were added, variations of the lattice constant would become very small.

In this paper, we will argue that the problem of finding the ground state of particles under constraints is equivalent to finding the particle distribution that is closer to a perfectly equilateral triangulation [17](the triangulation constructed from the actual distribution of particles via its Delaunay/Voronoi construction). The constraints that we consider in this paper are either geometrical (particles are constrained on spheres, torus, etc..) or topological (particles on a disk with the constraint that the total disclination charge is non-zero). If the particles are on a plane, the absolute ground state is a triangular lattice. The question is how the geometrical or topological constraints will modify this lattice.

The effect of the constraints is to induce spatial variations of the lattice constant, as it is sketched in figure 1for a square lattice. This is the same situation that was first addressed by Frank [18] in the context of crystal growth, where he showed that in order to minimize the strains induced by the variable spacing it was necessary to add additional rows of atoms, that is, dislocations, which correct the spatial variations in the lattice con- 
stant. Another critical result that we use in this paper is that the energy of particles interacting with a given potential can be regarded as an expression for large number of particles $M$ [12, 19], whose leading coefficient contains a term, the $C$-function, which is always positive and encodes the dependence on both geometry and topological defects. In this paper we will show how by adding dislocations it is possible to construct particle densities such that the $C$-coefficient becomes identically zero at leading order in $M$ (reaches its minimum value) thus providing explicit ground states for very large number of particles.

Although our arguments will be restricted to crystallization driven by energy, we believe that the geometric arguments used to construct ground states are general and apply to entropic driven crystallization such as for hard sphere potentials, since the entropic crystallization amounts to maximizing the area of the unit cell for a given packing fraction, and for larger packing fraction this leads to triangular lattices.

The organization of the paper is as follows. In section 1 we review several results regarding the expansion of the energy for large number of particles. In section III we implement the solution outlined in fig. 1 for a general triangular wedge, and it is shown in section IV that the ansatz does minimize the energy. Explicit results for the sphere and the torus are provided in section $\nabla$ We end with a summary of predictions that follow from this paper as well as with some conclusions in section DI.

\section{ENERGY IN THE LIMIT OF LARGE NUMBER OF PARTICLES}

The energy of $M$ particles interacting with a potential $V(\vec{r})$ is given by

$$
E(M)=\frac{1}{2} \sum_{i, j} V(\vec{r}(i)-\vec{r}(j))
$$

where the sum runs over all $M$ particles at positions $\vec{r}(i),(i=1 . . M)$. For definiteness, we discuss the concrete potential

$$
V(\vec{r})=\frac{e^{2}}{|\vec{r}|^{s}}
$$

but with minor modifications, the results can be made general to include any repulsive potentials. If the particles are arranged in a configuration close to a triangular lattice, we write $\vec{r}(i)=\vec{R}+\vec{u}(i)+\vec{h}(i)$, where $\vec{R}(i)=a\left(n e_{1}+m e_{2}\right)$ define the vertices of a triangular lattice of lattice constant $a$ and primitive vectors $e_{1}, e_{2}$, and $\vec{u}, \vec{h}$ are small quantities, in the sense that $\frac{|\vec{u}|}{a}<<1$. The quantity $u$ represents distortions tangent to the plane where $h$ in the perpendicular direction. The energy Eq. 1 is

$$
\begin{aligned}
E(M) & =\frac{M}{2} \sum_{n, m} \frac{e^{2}}{|\vec{R}(n, m)|^{s}}+\frac{e^{2}}{2} \sum_{i, j} \Pi_{\alpha \beta}(i, j) u_{\alpha}(i) u_{\beta}(j) \\
& \left.+\frac{e^{2}}{2} \sum_{i, j} \Pi^{0}(i, j) h(i) h(j)+\mathcal{O}\left(u^{3}, u^{2} h, h^{3}\right)\right)
\end{aligned}
$$

where many higher order terms are neglected because of the assumed smallness of the displacements $\vec{u}, h$. On a more rigorous basis, this step also requires the potential to be short ranged ( or $s>2$ ), thus excluding the Coulomb potential, although for some geometries such as the sphere, we expect Eq. 3 to hold as well, as it will be shown. In the above expression, the contributions related to the geometry and topological defects are entirely determined by the terms after the first.

We now review how the energy can be regarded as an expansion in large number of particles. Detailed derivations have already been presented somewhere else [12, 19], so we just recall the main results. To avoid excessive generality and keep the derivation simple, the results will be illustrated for the sphere. The first term in Eq. 3 gives the following explicit expression (for $0<s<2$ )

$$
\begin{aligned}
\frac{M}{2} \sum_{n, m} \frac{e^{2}}{|\vec{R}(n, m)|^{s}} & =\frac{e^{2}}{R^{s}}\left(\frac{M^{2}}{2^{s}(2-s)}+\right. \\
& \left.+\frac{\theta(s)}{2(4 \pi)^{s / 2}} M^{1+s / 2}+\mathcal{O}\left(M^{s / 2}\right)\right)
\end{aligned}
$$

where $R$ is the sphere radius. The only modification for $s>2$, is that the $M^{2}$ term, which arises from the long range nature of the potential, is absent. The actual values for the function $\theta(s)$ maybe found in [7, 19]. The second contribution in Eq. [3 is evaluated by retaining the leading term in an expansion in derivatives, leading to the familiar expression from elasticity theory

$$
\begin{aligned}
& \frac{e^{2}}{2} \sum_{i, j} \Pi_{\alpha \beta}(i, j) u_{\alpha}(i) u_{\beta}(j)+\frac{e^{2}}{2} \sum_{i, j} \Pi^{0}(i, j) h(i) h(j) \\
= & \int d^{2} \vec{r}\left(\mu u_{\alpha \beta}^{2}+\frac{\lambda}{2}\left(u_{\alpha \beta}\right)^{2}\right)
\end{aligned}
$$

where $u_{\alpha \beta}$ is the strain tensor and $\lambda, \mu$ are the Lame coefficients, whose explicit expression is ([19])

$\mu=\frac{\eta(s)}{(4 \pi)^{1+s / 2}} \frac{e^{2}}{R^{2+s}} M^{1+s / 2}, \quad \lambda=\frac{\varsigma(s)}{(4 \pi)^{1+s / 2}} \frac{e^{2}}{R^{2+s}} M^{1+s / 2}$

for $0<s<2, \varsigma=\infty$, but this is not important here (it becomes a constraint forcing the incompressibility of the crystal, but it has been shown that at leading order in $M$, the relevant elastic constant is the Young Modulus, which remains finite [19]). It should be recalled that Eq.[5 involves terms which are not quadratic, so some higher order terms have been included. Combining Eq. 5 and Eq. [6] it follows

$$
\int d^{2} \vec{r}\left(\mu u_{\alpha \beta}^{2}+\frac{\lambda}{2}\left(u_{\alpha \beta}\right)^{2}\right)=M^{1+s / 2} \frac{e^{2}}{R^{s+2}} C(s,[u])
$$


where the $C$-function is defined from

$$
C(s,[u])=\frac{1}{(4 \pi)^{1+s / 2}} \int d^{2} \vec{r}\left(\eta(s) u_{\alpha \beta}^{2}+\frac{\varsigma(s)}{2}\left(u_{\alpha \beta}\right)^{2}\right) .
$$

The $C$-function has dimensions of area. Combining Eq. 7 Eq. 5 and Eq. 4 the energy Eq. 3 becomes

$$
\begin{aligned}
E(M) & =\frac{e^{2}}{R^{s}}\left(\frac{M^{2}}{2^{s}(2-s)}+\left(\frac{\theta(s)}{2(4 \pi)^{s / 2}}+\frac{C(s,[u])}{R^{2}}\right) M^{1+s / 2}\right. \\
& \left.+\mathcal{O}\left(M^{s / 2}\right)\right)
\end{aligned}
$$

Let us now discuss the dependence of the $C$-coefficient on the sphere radius $R$. For any configuration $[u]$, we expect $C(s,[u]) \propto R^{2}$ and this implies that the energy at order $M^{1+s / 2}$ is increased with respect to the planar value Eq. 4. If, however, one could find a configuration $[u]$ such that its growth with $R$ is linear at most, then it follows that

$$
C(s, u) \propto R a \propto \frac{R^{2}}{\sqrt{M}}
$$

where $a$ is the lattice constant and in the last step, the relation $\frac{\sqrt{3}}{2} a^{2}=\frac{4 \pi R^{2}}{M}$ has been used. In this case, for very large number of particles, the coefficient $M^{1+s / 2}$ in the energy expansion is given by the planar result and the configuration $[u]$ becomes a minimum of the energy in the limit of large number of particles.

We now analyze the approximation made in ignoring higher derivative terms in Eq. 5 Those terms will consist in higher derivatives of the strain tensor, which in turn will imply that the elastic constants, equivalent to the Lame coefficients, contribute to terms growing more slowly than $M^{s / 2+1}$, that is, the ignored terms do not affect the leading term in the expansion defined by Eq. 9

In generalizing this expansion to other geometries there are several aspects to consider. First of all, the leading term for long-range potentials scales like $M^{2}$. The general expression of the coefficient is

$$
E^{0}=\frac{e^{2}}{2} \int d^{2} x d^{2} y \rho(x) \frac{1}{|x-y|^{s}} \rho(y)
$$

where $\rho(x)$ is the continuum density (The density of particles at scales much larger than a). In a sphere, $\rho$ is constant and given by $\rho=\frac{M}{4 \pi R^{2}}$, but in a general geometry, the density follows from minimizing the previous equation under the constraint $\int d^{2} x \rho(x)=M$ (For $s=1$ this amounts to solving the Poisson equation for a fixed density of charges). On a torus, for example, the resulting continuum density $\rho(x)$ is not constant for $s<2$. That is, the minimization of the leading coefficient for longrange potentials imposes density variations and invalidates the form of the coefficient $\mathcal{O}\left(M^{1+s / 2}\right)$ previously discussed. It is possible to generalize the expansion to include density variations but this will not be done here. On a general geometry, even for short-ranged potentials, the expansion Eq. 4 becomes slightly more complicated because there maybe more than one characteristic radius (In a torus, for example, there are two radii) and the coefficient $C$ will contain a dependence on the dimensionless parameters that can be constructed from the geometry (for the torus this is the aspect ratio, the ratio of the two radii), but the property defining the ground state configuration is still given by Eq. 10 The goal is now how to construct those configurations whose energy grows at most linearly with $R$.

\section{DISTRIBUTION OF DISLOCATIONS ON A TRIANGULAR WEDGE}

The problem consists now in obtaining the position and location of the dislocations needed to correct for the variable lattice constant, as discussed in the introduction. This will be obtained from the following geometric argument. Let us consider a triangular patch of a surface like the one shown in figure 2 At one vertex $(D$ in the figure), we place either a disclination of charge $q=1$ (a vertex with five nearest-neighbors), $q=0$ (a regular six fold vertex) or $q=-1$ (a vertex with seven nearest-neighbors).
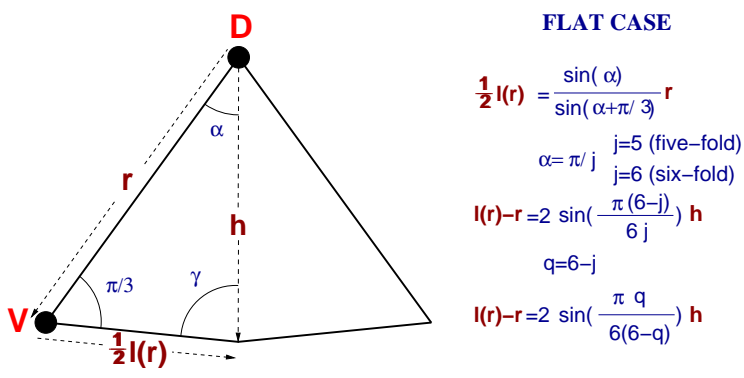

FIG. 2: (Color online) Triangle considered in the argument

If we assume that a disk is formed out of identical triangular wedges, the angle $2 \alpha$ is given by $2 \alpha=\frac{2 \pi}{6-q_{i}}$ ( for $q=+1,2 \alpha=\frac{2 \pi}{5}$ ). We now discuss the necessary conditions so that the wedge may be triangulated with many equilateral triangles. For that matter, we now compute by how much the length of segment $D V$, which is given by $r$, differs from the length of the segment whose origin is at $\mathrm{V}$ and forms 60 degrees with the segment $D V$ (because we assume that the triangle whose vertex is at $\mathrm{V}$ is equilateral). The quantity to compute is $l(r)-r$, which from the purely geometric arguments outlined in fig. 2 for the geometry of a plane is given by

$$
l(r)-r=2 \sin \left(\frac{q_{i} \pi}{6\left(6-q_{i}\right)}\right) h .
$$

if $l(r)-r=0$, which happens when $q_{i}=0$, the wedge can be tiled with all perfect equilateral triangles, but if the central disclination is non-zero, this is not possible. As explained in the introduction, we can fix this situation 
by including (or removing) additional rows of particles every time the equation

$$
l(r)-r= \pm a
$$

is satisfied, where $a$ is the lattice constant. In physical terms we are adding a dislocation. In the planar case, the formula above implies that we add a grain boundary of equally spaced dislocations, where dislocations within the grain are separated a distance $D=\frac{a}{2 \sin \left(\frac{q_{i} \pi}{6\left(6-q_{i}\right)}\right)}$. This result is well known in metallurgy [20], where grain boundaries of dislocations in the plane have been extensively investigated .

In an arbitrary geometry, the function $l(r)$ will also depend on the amount of Gaussian curvature enclosed within the triangular wedge, and this will lead to a different type of grain boundaries. The function $l(r)$ is now obtained from differential geometry,

$$
\begin{aligned}
l(r) & =\int_{-\frac{\pi}{m}}^{\varphi_{a}} d \varphi \sqrt{g_{a b}(\varphi) V^{a}(\varphi) V^{b}(\varphi)} \\
& +\int_{\varphi_{a}}^{\frac{\pi}{m}} d \varphi \sqrt{g_{a b}(\varphi) V^{a}(\varphi) V^{b}(\varphi)}
\end{aligned}
$$

where $g_{a b}$ is the metric of the geometry, $V^{a}$ is the tangent vector of a geodesic, described by coordinates $\varphi$, which starts at point $\left(r,-\frac{\pi}{m}\right)$, which is the equivalent of point $V$ in figure 2 and forms an angle of 60 degrees with the direction defined by the geodesic defined by the radial distance $r$. Once the function $l(r)$ is known, the location of the additional dislocations needed to ensure equilateral triangles will be obtained from the function $l(r)-r$ from Eq. 13] just as it was done for the planar case. In this paper, the distance $l(r)$ is computed from a geodesic, but in general this is not necessarily the case, and examples will be given when discussing the torus. It should be pointed out that the predictions that follow from Eq. 113 and Eq.12 are equivalent to a similar formula provided in 12. only in the plane, and differ on any other geometry.

The simplest non-trivial example involving curvature is a spherical cap. We introduce a new parameter $\theta_{M}$ that defines the angle subtended by the triangular wedge. We assume

$$
\theta_{M}=L \frac{a}{R}
$$

where $a$ is the lattice constant, $L$ the total number of particles along the radial direction $r$ and $R$ the radius of the sphere. We consider a spherical cap both with a $q=1$ disclination and without any disclination $q=0$ at its center, each case relevant for large and small aperture angle $\theta$ respectively.

The different functions $l(r)-r$ are shown in figure 3 for the cases of a five-fold $(q=1)$ and $(q=0)$ disclination and compared to the equivalent results for the plane. The grain boundaries that follow from the function above and Eq. 13 are shown in figure 4 for the particular situation $L=50$. In the figure, squares represent seven-fold and

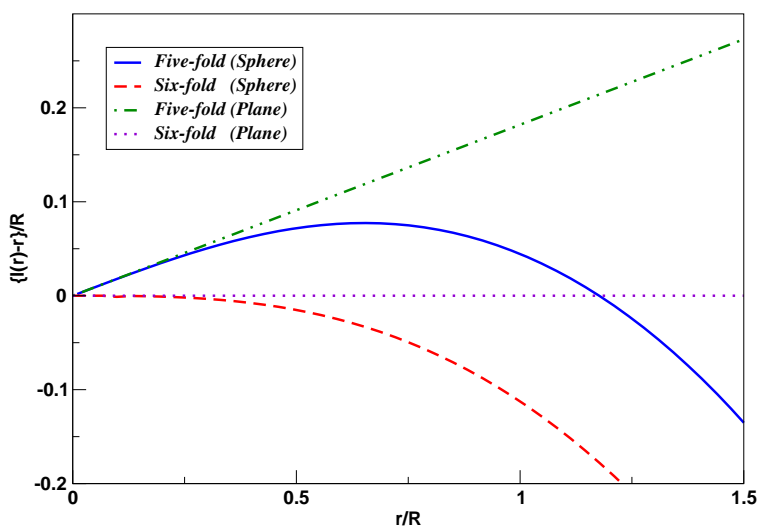

FIG. 3: (color online) $l(r)-r$ on a sphere for both $q=1,0$ and for a comparison, the results for the plane are also shown

circles represent five-fold vertices, with dislocations being represented as a five-seven pair. For large aperture angles the last dislocation has rotated 180 degrees, and this is a result of the function $l(r)-r$ hernmino neorative at $r / R$ lar

\section{Spherical cap $(L=50)$}

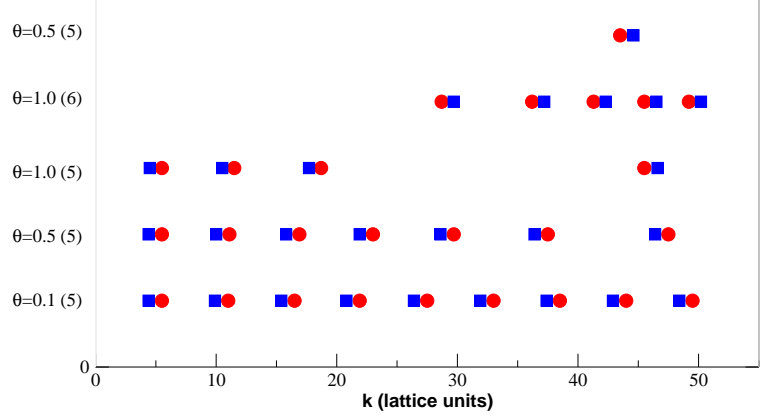

FIG. 4: (Color Online) Characteristic of grain boundaries of a spherical cap with different aperture angles with and without a disclination at the origin. Squares are seven-fold vertices and spheres are five-fold vertices. This plot corresponds to $L / a=50$.

Generalizations to any other geometry are now possible. As an example, we discuss a wedge of a torus. The critical difference with the sphere is that the Gaussian curvature in a torus is not constant, and that implies that the function $l(r)$ is different for the different wedges of a central defect. For a disk with a five-fold defect at its center, the five grain boundaries, which are identical for the sphere become now different. The second difference is that the line joining the dislocations is not straight, but curved, and depends on the torus aspect ratio

$$
r=\frac{R_{1}}{R_{2}},
$$


where $R_{1}$ and $R_{2}$ are the two torus radii. As an example the functions are shown for an aspect ratio $r=1.2$. In this case. as shown in fioure 5 onlv three of the functions $l 1$

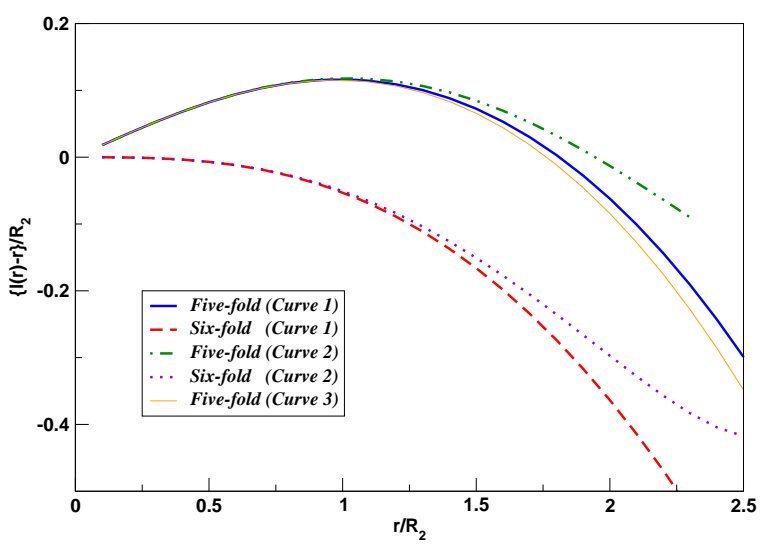

FIG. 5: (Color online) $l(r)-r$ on a torus at $r=1.2$ for both $q=1,0$. If the central defect is oriented as shown in figure 8 only three functions are needed for $q=1$ (fivefold). In this case $R_{1} / R_{2}=1.2$ and $\beta=0$.

\section{ENERGY OF OPTIMAL CONFIGURATIONS}

The previous argument provides the exact location where dislocations need to be added. Next, we compute the energy of these configurations. We will consider the energy for a large, yet finite number of particles. The elastic energy Eq. 5 can be discretized as 14]

$$
E_{1}(\varepsilon, \sigma)=\frac{\varepsilon}{2} \sum_{(b, c)}\left(\left|\vec{r}_{b c}\right|-a\right)^{2}+\sigma \sum_{<f, d>}\left(\frac{1}{2}-\cos \left(\theta_{d f}\right)\right)^{2} .
$$

where $(b, c)$ runs over edges defined by nearest neighbors and angles. This energy has a very clear geometric interpretation as providing an energy cost for those triangles that either are formed of edges whose length is not the lattice constant, or whose angles are not exactly $60^{\circ}$. The coefficients $\varepsilon, \sigma$ are linearly related to the Lame coefficients [14, 21].

We now evaluate the energy function Eq.17] in the case $\sigma=0$ for the wedge discussed in the previous section. By construction, the length of the edges along the radial distances $r$ are exactly given by $a$, the lattice constant, and the contribution to the energy of these edges is zero, consistent with the total radial length of the wedge given by $H=L a$, where $a$ is the lattice constant and $L$ the total number of edges. The main contribution to the energy then comes from the vertices along the direction defined by $l(r)$. At radial distance $r=k a$ there are $n(k)$ of these vertices, and therefore, the average length of nearest neighbors along the radial distance $k a$ is $l(k a) / n(k)$.
The energy Eq. 17 becomes

$$
E_{1}(\varepsilon)=\frac{\varepsilon}{2} \sum_{k=1}^{L} \frac{(l(k a)-n(k) a)^{2}}{n(k)}+N_{d} E_{\text {elas }} .
$$

The second term is proportional to the total number of dislocations $N_{d}$ and takes into account that next to a dislocation the strains are significant. This energy includes not only the core energy but also the (exponentially) small distortions arising from the grains. At this point it is illustrative to show the implications of the previous formula for a simple and well known situation. We now compute the energy of a disk consisting of no defects other than a central disclination at the origin. In that case $l(r)=b r$, where $b$ is given from figure 2] and since no additional dislocations are added it is $n(r=k a)=k$. The above formula gives (with $N_{d}=0$ )

$$
E_{1}(\varepsilon)=5 \frac{\varepsilon}{2} \int_{0}^{R} \frac{d r}{r}(l(r)-r)^{2}=\frac{5 \varepsilon}{4}(b a)^{2} L^{2}
$$

as expected, the energy of an isolated disclination grows quadratically with $L^{2}$. Thus the $C$-coefficient defined by Eq. [8] will grow like $H^{2}$, and lead to additional contributions to Eq. 9 (here $H=L a$ plays the role of $R$ in the sphere). All these results are well known [14, 21] but it is of interest to show how they are recovered within the previous approach. It is now easy to show that the energy of the configurations that satisfy Eq. 13 grow more slowly than $R$. We have,

$$
\begin{aligned}
E_{1}(\varepsilon) & =\frac{\varepsilon}{2} \sum_{k=1}^{L} \frac{(l(k a)-n(k) a)^{2}}{n(k)} \\
& <\frac{\varepsilon}{2} \sum_{k=1}^{L} \frac{1}{n(k)} a^{2} \sim \log (L)
\end{aligned}
$$

where the last step follows because by construction $l(r)-n(r) a$ never exceeds one. Within the same assumptions, it is very simple to repeat the argument for the second term in Eq. 17 and the same logarithmic behavior is found, so the statement holds for the entire range of elastic constants. As already mentioned, distortions near dislocations are not entirely negligible, and this leads to a term that grows linearly with $L$, .

$$
E_{1}(\varepsilon)=N_{d} E_{\text {core }} \sim H a=L a^{2} .
$$

As an example of the previous considerations, we discuss the spherical cap. We determine best distributions as a function of $\theta_{M}$, the subtended angle, and $L$ the radial number of particles. As $\theta_{M} \rightarrow 0$, the spherical cap becomes a plane and the ground state should approach the energy of a perfect planar lattice. As $\theta_{M}$ is increased, Gaussian curvature effects become important, and a disclination at the top of the cap lowers the energy as shown in the inset of fig. 6] Upon including dislocations the energy decreases enormously and remains essentially independent with the subtended angle in both cases. 


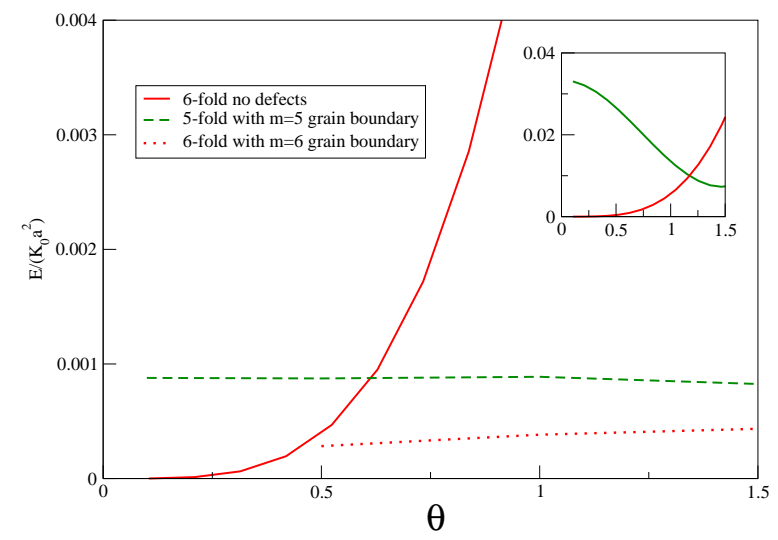

FIG. 6: (Color Online) Energy of the spherical cap $(\mathrm{L}=50)$ with and without defects. The contribution proportional to the number of dislocations is not shown.

The previous considerations do show that the geometrical ansatz does provide a minimum energy configurations, albeit a degenerate one. For the spherical cap, for example, the minimum energy can be achieved either by a plus disclination or without any disclinations at all, provided that the appropriate grain boundaries, as defined by Eq. 13 are used. The next question is therefore, which one of these minima is the actual ground state of the system. This involves considering the sub-leading coefficients in the expansion. From Eq. [20] this is given by the configuration with the lowest possible number of defects. This still does not completely solve the problem. One can consider several grain boundary on each triangular wedge where the separations of dislocations within the grain is larger, thus keeping the total number of defects constant. For the planar case, this question was investigated in [14] where it was concluded that grain boundaries with the smallest spacing within dislocations are favored. Further numerical investigations will hopefully provide more evidence on this point.

\section{SOLUTIONS FOR THE SPHERE AND THE TORUS}

\section{A. The Sphere}

There are several topological inequivalent triangulations of a sphere with icosahedral symmetry, and are labelled by two integers $(n, m)$. We just describe here in detail the solutions to the cases $(n, n)$ and $(n, 0)$, but solutions of the form $(n, m)$ may be constructed along the same lines. The problem consists now in dividing the sphere into triangular wedges such that can be consistently joined back together after the additional dislocations necessary to relieve the geometric frustration have been included.

The $(n, n)$ configuration can be divided into 20 trian- gular wedges like the one shown in fig. 7 (2 triangular wedges are shown). From the results described for spherical wedges, the dislocations follow the line $\mathrm{AB}$, and the spacing is predicted from the function fig. [3 for an aperture angle $\theta_{M} \equiv \theta_{V}=\arcsin (2 \sqrt{1 / 2-1 /(2 \sqrt{5})} / \sqrt{3}) \approx$ $37^{\circ}$. The fact that the angle $\mathrm{ABC}$ is 90 degrees and the angle ACB is 60 degrees, which can be checked by using formulas in spherical trigonometry, ensures that neighboring triangular wedges are perfectly joined and the complete consistency of the solution.

The triangular wedges for the $(n, 0)$ are shown in fig. [ 7 A natural patch is defined by DCO, but it should be noted that $\mathrm{CO}$ does not define a row of particles of the crystal. In this case, the patches extend beyond point C, and overlap slightly. The dislocations follow the lines DO and the spacing is predicted from the results from the function fig. 3 similarly as in the $(n, n)$ case. Here, however, the consistency is a little more difficult to check, because in the region defined by such triangles such as COP, which spans aperture angles between $\left(\theta_{G}, \theta_{V}\right)$, where $\theta_{M} \equiv \theta_{G}=\arccos (1 / \sqrt{(5)}) / 2 \approx 32^{\circ}$, the different patches overlap. The consistency here is due to the sum of the Burgers vectors of the three grain boundaries that approach point $\mathrm{O}$ adding to zero, which ensures that all rows of particles added terminate within the patches.

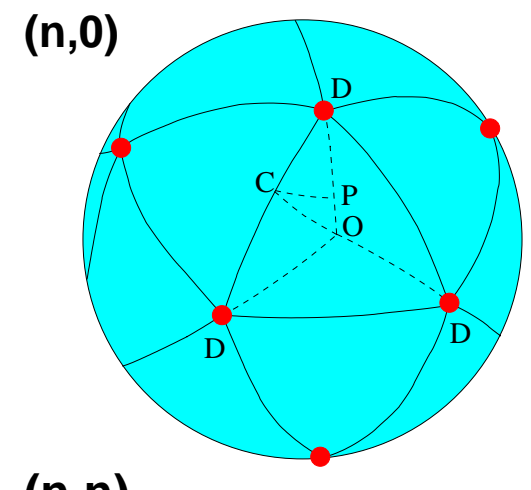

$(n, n)$

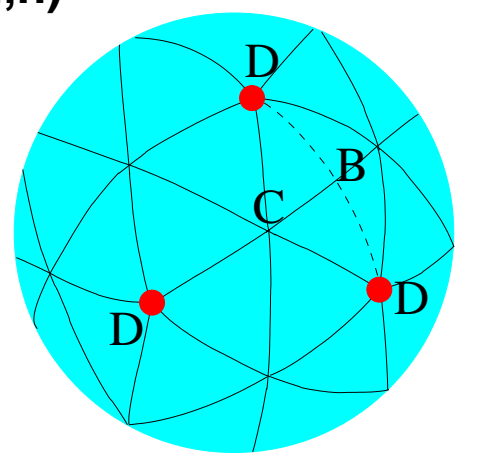

FIG. 7: (Color Online)Triangular wedges used to solve the $(n, n)$ and the $(n, 0)$ configurations. Dashed lines follow the directions of the grain boundaries. Filled circles represent + disclinations, also marked with D. 


\section{B. The Torus}

The torus is depicted in fig. 8 There are two radii curvature, $R_{1}, R_{2}$, and the aspect ratio is defined by $r=\frac{R_{1}}{R_{2}}>1$. As already discussed the only situation we discuss concerns short-ranged potentials. The Gaussian curvature of the torus depends only on the coordinate $\varphi$, and it is

$$
K=\frac{\cos (\varphi)}{R_{2}^{2}(r+\cos (\varphi))}
$$

Since Gaussian curvature attracts like sign disclinations [6], we assume here that the ground state of the torus contains 12 positive disclinations located along the geodesic of maximum curvature (outer curve) and 12 negative disclinations along the geodesic of minimum curvature (inner curve), and several grain boundaries of dislocations. Another possibility would consist of a ground state consisting of grain boundaries of dislocations only. It is very likely that for a thin torus $r>>1$ disclinations may not be favored because nearest-neighbor disclinations are so far away, that radial grain boundaries may not efficiently screen the strains.

If the ground state contains disclinations, the grain boundaries that appear following the five-fold disclinations have already been computed in figure 5. There is a similar function for the seven-fold disclinations, which are located along the interior circle in the figure. The function predicting its spacing is given in figure 9

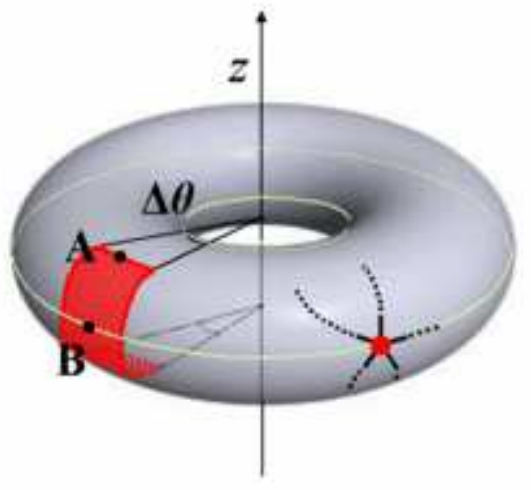

FIG. 8: (Color Online)Representation of the torus, with the definition of the subtended angle. The two circles are geodesics of maximum and minimum curvature. A fivefold defect on the positive curvature of the torus has been shown.

The situation were the ground state of the torus consists of grain boundaries of dislocations only is qualitative different, because in that case, the grain boundaries are not radial, but follow the directions defined by the azimuthal angle, the curve $A B$ in figure 8 and a similar curve with opposite oriented dislocations on the inner

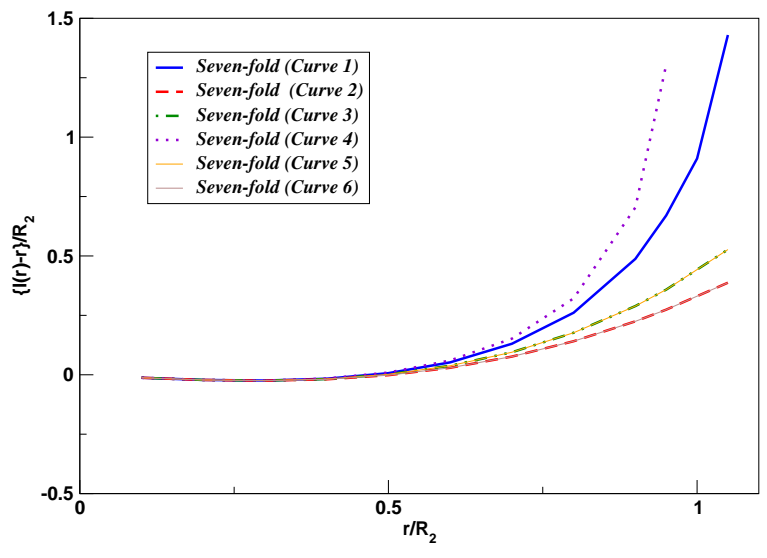

FIG. 9: (Color Online) $l(r)-r$ on a torus at $r=1.2$ for both $q=1$. The different functions are shown. $\left(R_{1} / R_{2}=1.2\right.$ and $\beta=0)$

side of the torus. The particles are located in rings defined by the condition $\psi=$ constant, and therefore, only $\psi=0, \pi$ are geodesics, which correspond to the two circles in fig. 9] Similarly as in the sphere, there are other torus triangulations that maybe constructed from twisting and rejoining the lattice either along the varphi or the $\theta$ direction, but the discussion of the grain boundaries in this case is beyond the scope of this paper.

\section{CONCLUSIONS}

We now summarize some of the main predictions that follow from the results presented in this paper that can be verified from either numerical simulations or experimental results:

1. The ground state consists of grain boundaries of dislocations whose exact position and orientation is predicted from Eq. 13] Explicit examples have been provided for both the sphere and the torus.

2. The Energy of the ground state tends to a universal value for very large number of particles, which is independent of the geometry and given by Eq. 4 (For long-range potentials, there is an additional term, which grows quadratically with the number of particles).

3. For potentials $V(r)=\frac{e^{2}}{r^{s}}$, the sub-leading corrections to the ground state are of order $M^{(s+1) / 2}$ in the number of particles.

4. The ground state is degenerate at leading order, as there are some free parameters characterizing the grain boundaries. This degeneracy is removed at sub-leading order by those configurations with the minimum number of dislocations and the lowest possible number of grain boundaries. 
There are several ways to verify these predictions. The main difficulty with numerical minimizations is that it is very difficult to prepare an initial configuration that will relax to a previously selected distribution of defects. For the Thomson problem, however, the ring-removal technique developed by Toomre [22] seems to generate the type of grain boundaries that this paper predicts as the ground state. In fact, in 23], it was shown that these grain boundaries significantly lower the energy, bringing the icosadeltahedral configurations closer to the planar limit Eq. 14 It was further speculated that the true ground state could be achieved by successive applications of the technique, but this statement was not substantiated because the actual rings that needed to be removed were not known. The function in figure 3 together with Eq. 13] does provide the location of the dislocations and therefore the actual rings that need to be removed, so the ring removal technique appears as a very promising practical tool to verify predictions 1 to 3 for the Thomson problem. It would also be of great interest to check how this predictions compare with ground states for shortranged potentials, where the results of this paper are more rigorously justified.

Evidence on the validity of statements 1-4 has also been provided from numerical and analytical results in [14], where by using a discretized version of elasticity theory, it is shown that 1-4 are verified for a plane with the constraint of total disclination charge equal to \pm 1 . The methods presented, however, are far general and it is expected that the extension of the results for any geometry will soon follow.

Statement 2) is in agreement with recent rigorous results proven for potentials $s>2$ [7, 8]. These results have greater generality, since they apply to dimensions other than 2. Preliminary results for the torus with shortranged potentials have recently become available 24, 25] and show density variations for $s<2$ and ground states for $s>2$ with and without disclinations, but the present state of the simulations do not allow to draw a more quantitative analysis. Experimental evidence can also be used to prove the validity of statement 1 . In [5] it was shown that PMMA beads assemble on an spherical oil-water interface forming spherical crystals, which can then be imaged by confocal microscopy. The current experiments show that next to five-fold defects additional dislocations arise, but there are only two of them, instead of the five predicted by this paper (Interestingly this is the number favored in the flat case [14]). Furthermore, the dislocations within the grain show a constant spacing. This is possibly due to the fact that the total number of particles is still too small for the asymptotic results of this paper to apply. Future experiments with larger particle aggregation numbers should settle this issue.

An important point that has not been addressed in this paper is the critical value of $M$ at which the asymptotic solution proposed will apply. A numerical verification of the predictions of this paper is currently under way for short-ranged potentials and preliminary results indicate that for the sphere, this number is of the order of five thousand particles at the very least. We hope to report more on this in the near future.

In applying the formulas derived to a real situation, the dislocations must be located at points in the crystal, and accordingly, the spacing of the dislocations must always be an integer. To apply the previous formulas, the location of the dislocations must therefore be rounded to the closer integer. The resulting orientations of the dislocations may also not be entirely consistent with the location of the crystallographic axis of the crystal, and this may require, for example, placing dislocations separated by an odd number of lattice constants 14]. Further numerical work will hopefully clarify this more technical points.

There are a certain number of issues that this paper has not addressed. First of all, the ground states discussed for both the sphere and the torus rigorously apply only for some "magic" number of particles $M$. We expect that for large number of particles outside this magic numbers, the ground state solutions should not be that different from the ones proposed in this paper, since the range of $M$ in between magic numbers is small compared with $M$ itself. We hope that future numerical work will address this issue.

In this paper, it has been assumed that the potential is isotropic. If the potential is not isotropic the ground state on a plane may not be a triangular lattice, and the present arguments need to be modified. For very large number of particles, it should be expected that locally, the triangulation will be very close to the flat case, so similarly as in the isotropic case, we expect that additional dislocations will be required to fix the frustration induced by the geometric constraints.

There has been recent interest in understanding similar problems as the one discussed in this paper where liquid crystalline order is discussed on a non-zero geometry. Examples include hexatic [26, 27, 28], nematic 29], or smectic blue phases [30]. The results discussed in this paper, where the energy is regarded as an expansion in $M$ and the defects correct for the geometric frustration are completely general and may be generalized to this cases as well. We hope to report more in the near future.

\section{Acknowledgments}

This paper has arisen from many discussions with Mark Bowick and David Nelson. Discussions with Angelo Cacciuto,Slava Chushak, Doug Hardin and Edward Saff are also acknowledged. This work has been supported by NSF grant DMR-0426597 and by Iowa State Start-up funds. 
[1] J.H. Conway and N.J.A. Sloane. Sphere Packings, Lattices and Groups. Springer-Verlag, New York, 2nd edition, 1993.

[2] E.B. Saff and A.B. Kuijlaars. Mathem. Intell., 19:5, 1997.

[3] J.J. Thomson. Philos. Mag., 7:237, 1904.

[4] U Albrecht and P. Leiderer. J. Low Temp. Phys., 86:131, 1992.

[5] A.R. Bausch, M. Bowick, A. Cacciuto, A. Disnmore, M. Hsu, D.R. Nelson, M. Nikolaides, A. Travesset, and D. Weitz. Science, 299:1716, 2003.

[6] M. Bowick, D.R. Nelson, and A. Travesset. Phys. Rev. $B, 62: 8738,2000$.

[7] D.P. Hardin and E.B. Saff. Notices of Am. Math. Soc., 51:1186, 2004.

[8] D.P. Hardin and E.B. Saff. Adv. in Math.,193:174, 2005.

[9] E.L. Altschuler, E.R. Williams, R. Ratner, R. Tipton, R. Stong, and F. Wooten. Phys. Rev. Lett., 78:237, 1997.

[10] D.M. Deaven, N. Tit, J.R. Morris, and K.M. Ho. Chemical Physics Letters, 256:195, 1996.

[11] T. Erber and G.M. Hockney. Advances in Chemical Physics, 98:594, 1997.

[12] M. Bowick, A. Cacciuto, D. Nelson, and A. Travesset. Phys. Rev. Lett., 89:185502, 2002.

[13] M. Bowick and A. Travesset. J. Phys. A:Math. Gen. 34 (2001) 1, 2001.

[14] A. Travesset. Phys. Rev. B, 68:115421, 2003.
[15] M. J. .W. Dodgson and M.A. Moore. Phys. Rev. B, 51:11887, 1995.

[16] A. Perez-Garrido, M. J. .W. Dodgson and M.A. Moore. Phys. Rev. B, 56:3640, 1997.

[17] We are assuming isotropic potentials. If the potential is not isotropic, in that case the ground state in the plane may be different than the triangular lattice.

[18] Frank. Z. Elektrochem., 56:337, 1952.

[19] M. Bowick, D. Nelson, and A. Travesset. preprint, 2005.

[20] J. Hirth and J. Lothe "Theory of Dislocations", 1992, Krieger Publishing Company.

[21] S. Seung and D. R. Nelson. Phys. Rev. A, 38:1005, 1988.

[22] A. Toomre. Unpublished, 1997.

[23] A. Perez-Garrido and M.A. Moore. Phys. Rev. B, 60:15628, 1999.

[24] M. Bowick, C. Cecka, and A. Middleton. http://www.cs.hmc.edu/ ccecka, 2005.

[25] R. Womersley. www.maths.unsw.edu.au/ rsw, 2005.

[26] M. Bowick, D.R. Nelson, and A. Travesset. Phys. Rev. E, 69:41102, 2004.

[27] V. Vitelli and D. Nelson. Phys. Rev. E, 70:51105, 2004.

[28] V. Vitelli and A.M. Turner. Phys. Rev. Lett., 93:215301, 2004.

[29] D. Nelson. Nano Letters, 2:1125, 2002.

[30] B. DiDonna and R. Kamien Phys. Rev. E, 68:41703, 2003. 